\title{
Indicadores morfométricos em cabeças de espécies brasileiras de Chrysoperla (Neuroptera, Chrysopidae)
}

\author{
Sérgio de Freitas ${ }^{1} \&$ Adriana Coletto Morales ${ }^{1}$
}

${ }^{1}$ Departamento de Fitossanidade, Faculdade Estadual Paulista, Universidade de Ciências Agrárias e Veterinárias. Rodovia Paulo Donato Castellane, s/nº.14884-900 Jaboticabal-SP. Brasil.serfre@fcav.unesp.br; morales@rge.fmrp.usp.br

\begin{abstract}
Morphometric studies of the head of Brazilian species of Chrysoperla (Neuroptera, Chrysopidae). The species C. externa, C. defreitasi, C. raimundoi and C. genanigra (Neuroptera, Chrysopidae) have great differences in the genitalia. These species were studied morphometrically by the distances between points of the head (eight linear measurements) to better identify them. The results, analyzed by multivariate statistics, showed that it is possible to identify measures that distinguish the individual, although some species are more distinguishable by morphometry. The canonical analysis showed that three of the four species, C. raimundoi, C. genanigra and C. externa, showed no major overlap between them, and their possible discrimination within the group, only $C$. defreitasi presented a low resolution according to this analysis, being overlapped with $C$. externa and $C$. genanigra, however, it is totally broken in $C$. raimundoi. The measures that contributed most to differentiation are related to the size of the head and especially the length of wrasse on the edge of the head, until the level of the eyes and antenna.
\end{abstract}

KEYWORDS. Biological control; cryptic species; morphometrics; predator.

\begin{abstract}
RESUMO. Indicadores morfométricos em cabeças de espécies brasileiras de Chrysoperla (Neuroptera, Chrysopidae). As espécies do gênero Chrysoperla que ocorrem no Brasil -C. externa (Hagen, 1861), C. defreitasi (Brooks, 1994), C. raimundoi (Freitas \& Penny, 2001) e C. genanigra (Freitas, 2003)-, que apresentam as principais diferenças nas genitálias, foram estudadas morfometricamente através das distâncias entre pontos da cabeça (oito medidas lineares) a fim de melhor identificá-las. Os resultados, analisados através da estatística multivariada, mostraram que é possível se identificarem medidas que as distinguem individualmente, muito embora algumas espécies sejam mais distinguíves através da morfometria. A análise canônica mostrou que três das quatro espécies, $C$. raimundoi; $C$. genanigra e $C$. externa, não apresentaram grandes sobreposições entre si, sendo possível sua discriminação dentro do grupo, somente $C$. defreitasi apresentou uma baixa resolução de acordo com esta análise, ficando sobreposta com C. externa e C. genanigra, no entanto, ela é totalmente discriminada de C. raimundoi. As medidas que mais contribuíram para a diferenciação estão relacionadas ao tamanho da cabeça e em especial ao comprimento do labro, na extremidade da cabeça, até o nível dos olhos e antena.
\end{abstract}

PALAVRAS-CHAVE. Controle biológico; espécies crípticas; morfometria; predador.

O controle biológico de pragas vem sendo utilizado cada vez mais como método eficiente de limitação populacional de espécies nocivas à agricultura. Apesar de os entomopatógenos e parasitóides se destacarem entre os diversos agentes que têm sido utilizados nos programas, no Brasil, observa-se um crescente interesse em relação aos predadores, principalmente sobre os crisopídeos. Essa mesma tendência tem sido observada em outros países como Estados Unidos onde esse grupo de insetos é de grande importância (Stelzl \& Devetak 1999; Tauber et al. 2000).

Dentre os predadores destacam-se as espécies de Chrysopidae, uma grande família da Ordem Neuroptera que inclui mais de 1200 espécies e subespécies distribuídas entre 86 gêneros e subgêneros (Brooks \& Barnard 1990). Nela estão incluídos insetos que apresentam as seguintes características: tamanho médio de 10 a $15 \mathrm{~mm}$ de comprimento; coloração variando do verde claro, amarelo e até marrom; olhos escuros ou dourados, antenas filiformes; asas hialinas com ou sem manchas escuras, apresentando nervuras em rede (Adams \& Penny 1985).
O gênero Chrysoperla, um dos mais importantes, possui 36 espécies, das quais quatro podem ser encontradas no Brasil: C. externa (Hagen, 1861), C. defreitasi (Brooks, 1994), C. raimundoi (Freitas \& Penny, 2001) e C. genanigra (Freitas, 2003). Destas, C. externa se destaca por apresentar uma ampla distribuição geográfica, sendo encontrada nos Estados Unidos (Flórida, Carolina do Sul e Texas), México, Guatemala, Honduras, Cuba, Haiti, Colômbia, Venezuela, Equador, Peru, Paraguai, Chile e Argentina (Brooks 1994).

Essas espécies são muito próximas entre si, e observações na morfologia externa têm revelado variações nas diferentes populações. Por isso, a determinação do grau de variação se faz necessária para que sejam estabelecidas características com possibilidade de serem utilizadas como caracteres para o reconhecimento e identificação das espécies.

Já se encontra estabelecido que para que haja sucesso em programas de controle biológico, é fundamental uma correta identificação específica. Entretanto, são comuns os equívocos no momento das determinações, geralmente decorrentes do 
desconhecimento da variação morfológica intraespecífica.

Em relação a essas quatro espécies de Chrysoperla, as principais características que são compartilhadas e que podem levar a uma identificação incorreta são: antenas claras na base e escuras no ápice; corpo verde com faixa amarela longitudinal no dorso do tórax e abdome. Já as características que as diferenciam são: fronte com ou sem marcas vermelhas próximas às genas; genas vermelhas ou pretas; asas hialinas, com ou sem transversais pretas (Hagen 1861; Brooks 1994; Freitas \& Penny 2001; Freitas 2003). Embora haja essas características que as diferenciam, muitas vezes, elas não são suficientes para a completa identificação deste grupo de espécies.

Este estudo tem por objetivo minimizar os problemas relacionados ao reconhecimento das espécies crípticas $C$. defreitasi, C. externa, C. raimundoi e C. genanigra, através da identificação de parâmetros que, associados a outros aspectos, ressaltem as diferenças individuais em relação às características morfológicas externas da cabeça.

\section{MATERIALE MÉTODOS}

Através de uma análise morfométrica determinou-se a variação morfológica cefálica, utilizando-se para isto uma análise discriminante, a qual fornece a descrição da diferença entre grupos especificados a priori, em um conjunto de dados multivariados. Essa análise se propõe a verificar a magnitude de diferença entre os grupos relativa àquela dentro dos grupos e qual a significância estatística desta magnitude.

Adultos das quatro espécies pertencentes à coleção do Laboratório de Biossistemática de Crisopídeos da Faculdade de Ciências Agrárias e Veterinárias (FCAV-UNESP), Campus de Jaboticabal, Estado de São Paulo, foram estudados. Os exemplares de C. externa, $C$. defreitasi e $C$. raimundoi foram coletados em Jaboticabal e $C$. genanigra foram coletados em Mossoró, Estado do Rio Grande do Norte. Foram utilizados somente indivíduos machos, sendo 42 indivíduos da espécie Chysoperla externa; 36 da C. defreitasi; 21 da C. genanigra e 30 da espécie $C$. raimundoi.

As mensurações foram obtidas a partir de oito variáveis

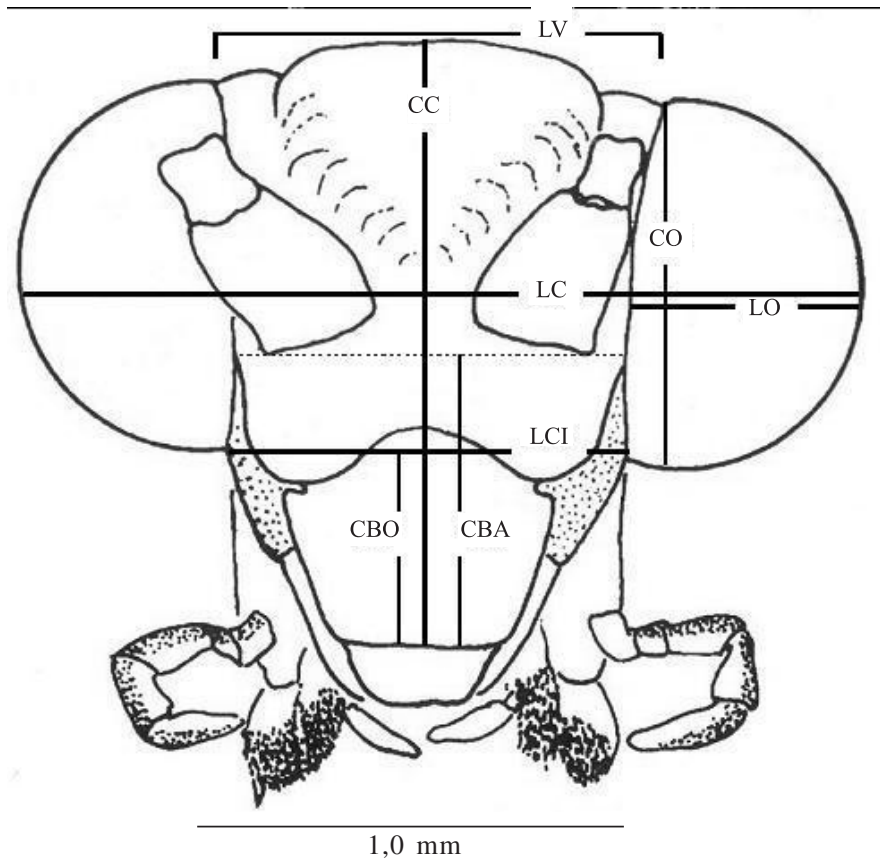

Fig. 1. Medidas representativas para a análise morfométrica realizada na cabeça de quatro espécies brasileiras de Chrysoperla (Neuroptera, Chrysopidae). CC - comprimento; LC - largura; LV - largura do vértice; LCI - largura da cabeça ao nível da base dos olhos; CBO - comprimento até a base dos olhos; CBA - comprimento do labro à base das antenas; $\mathrm{CO}$ - comprimento do olho e LO - largura do olho.

morfométricas da cabeça de cada indivíduo (Fig. 1): comprimento da cabeça (CC); largura da cabeça (LC); largura do vértice (LV); largura da cabeça na base dos olhos (LCI); comprimento do labro à base dos olhos $(\mathrm{CBO})$; comprimento do labro à base das antenas (CBA); comprimento do olho (CO) e largura do olho (LO).

As cabeças foram fotografadas em vista frontal, com auxílio de câmera acoplada ao estereomicroscópio, no ponto em que a extremidade do labro e do vértice estavam em foco. As mensurações foram realizadas com auxílio da câmera MOTICAM e software IMAGE da Motic.

Tabela I. Média (m), moda (md), variância (var) e a amplitude da variação em milímetros (ampl), para cada uma das variáveis para a cabeça de Chrysoperla raimundoi (Freitas \& Penny, 2001), C. defreitasi (Brooks, 1994), C. genanigra (Freitas, 2003) e C. externa (Hagen, 1861) (Neuroptera, Chrysopidae).

\begin{tabular}{|c|c|c|c|c|c|c|c|c|c|c|c|c|c|c|c|c|}
\hline & \multicolumn{4}{|c|}{$\begin{array}{c}\text { C raimundoi } \\
\mathrm{n}=30\end{array}$} & \multicolumn{4}{|c|}{$\begin{array}{c}\text { C. defreitasi } \\
\mathrm{n}=36\end{array}$} & \multicolumn{4}{|c|}{$\begin{array}{c}\text { C. externa } \\
\mathrm{n}=42\end{array}$} & \multicolumn{4}{|c|}{$\begin{array}{c}\text { C. genanigra } \\
\mathrm{n}=21\end{array}$} \\
\hline & $\mathrm{m}$ & md & var & ampl & $\mathrm{m}$ & md & var & ampl & $\mathrm{m}$ & md & var & ampl & $\mathrm{m}$ & md & var & ampl \\
\hline $\mathrm{CC}$ & 1,1 & 1,1 & 0,005 & $1,0-1,2$ & 1,03 & 1,0 & 0,009 & $0,7-1,2$ & 1,05 & 1,0 & 0,004 & $1,0-1,2$ & 0,83 & 0,9 & 0,007 & $0,7-1,0$ \\
\hline LC & 1,5 & 1,5 & 0,008 & $1,4-1,9$ & 1,42 & 1,4 & 0,008 & $1,2-1,6$ & 1,40 & 1,4 & 0,002 & $1,3-1,5$ & 1,25 & 1,2 & 0,017 & $1,1-1,5$ \\
\hline LV & 0,81 & 0,8 & 0,004 & $0,7-0,9$ & 0,78 & 0,8 & 0,003 & $0,7-0,9$ & 0,71 & 0,7 & 0,002 & $0,6-0,8$ & 0,70 & 0,7 & 0,005 & $0,6-0,9$ \\
\hline LCI & 0,72 & 0,7 & 0,002 & $0,6-0,9$ & 0,65 & 0,7 & 0,003 & $0,5-0,7$ & 0,60 & 0,6 & 0,000 & $0,5-0,7$ & 0,57 & 0,6 & 0,002 & $0,5-0,6$ \\
\hline $\mathrm{CBO}$ & 0,41 & 0,4 & 0,002 & $0,3-0,5$ & 0,30 & 0,3 & 0,003 & $0,1-0,4$ & 0,31 & 0,3 & 0,001 & $0,2-0,4$ & 0,20 & 0,2 & 0,003 & $0,1-0,3$ \\
\hline CBA & 0,72 & 0,7 & 0,002 & $0,6-0,8$ & 0,49 & 0,5 & 0,005 & $0,3-0,6$ & 0,45 & 0,5 & 0,004 & $0,3-0,6$ & 0,39 & 0,4 & 0,003 & $0,3-0,5$ \\
\hline $\mathrm{LO}$ & 0,68 & 0,4 & 0,001 & $0,3-0,5$ & 0,62 & 0,4 & 0,002 & $0,3-0,4$ & 0,64 & 0,4 & 0,001 & $0,3-0,4$ & 0,54 & 0,3 & 0,004 & $0,2-0,4$ \\
\hline $\mathrm{CO}$ & 0,39 & 0,7 & 0,001 & $0,6-0,7$ & 0,36 & 0,6 & 0,002 & $0,5-0,7$ & 0,38 & 0,6 & 0,002 & $0,6-0,7$ & 0,31 & 0,5 & 0,003 & $0,5-0,7$ \\
\hline
\end{tabular}

CC - comprimento da cabeça; LC - largura; LV - largura do vértice; LCI - largura da cabeça ao nível da base dos olhos; CBA - comprimento até a base das antenas; $\mathrm{CBO}$ - comprimento até a base dos olhos; CO - comprimento dos olhos e LO - largura dos olhos. 


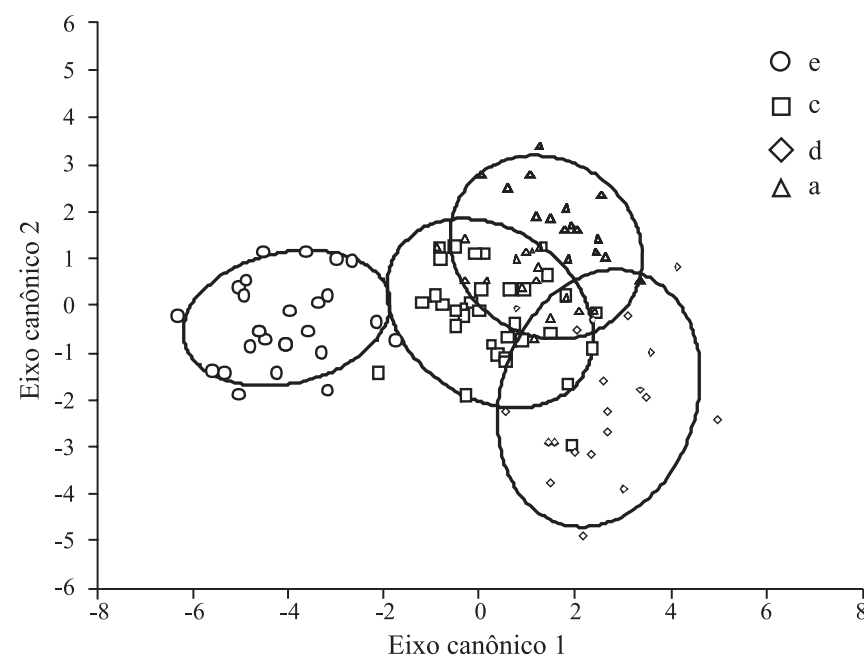

Fig. 2. Discriminação das quatro espécies de Chrysoperla (Neuroptera, Chrysopidae) estudadas: (e) - C. raimundoi (Freitas \& Penny, 2001); (c) - C. defreitasi (Brooks, 1994); (d) - C. genanigra (Freitas, 2003) e (a) - C. externa (Hagen, 1861), baseada nos dois primeiros eixos canônicos.

Para as análises estatísticas cada espécie representou um grupo. As médias e o desvio padrão para cada grupo foram calculados através das oito variáveis morfológicas. Também foram determinadas a moda e a variância para cada característica, o que permitiu diagnosticar a amplitude da variação para cada uma das espécies estudadas. One-Way ANOVA foi usada para comparações médias. A análise de função discriminante stepwise foi utilizada para se predizer que variáveis efetivamente contribuem para a discriminação entre os grupos, neste caso as quatro espécies. A significância de Wilks' lambda foi utilizada para se determinar o grau em que medidas distintas contribuíram para o modelo final de discriminação.

A análise canônica também foi empregada para se acessar as relações entre as variáveis. $\mathrm{O}$ valor absoluto dos coeficientes canônicos obtidos da análise de variáveis canônicas representa o peso de cada variável na formação da raiz canônica, onde altos valores correspondem a maior diferenciação entre os grupos. Todos esses parâmetros estatísticos foram calculados pelo programa Statistica v. 6. StatSoft, Inc. (2001). A análise de distância de Mahalanobis foi realizada utilizandose o programa Mega versão 3.1 (Kumar et al. 2004).

\section{RESULTADOS EDISCUSSÃO}

Através das oito medidas obtidas linearmente foi possível estimar os valores médios, a moda e a variância para cada uma das medidas da cabeça (Tabela I); e ainda, a razão entre o comprimento e largura da cabeça; razão entre o comprimento da distância do labro até a base dos olhos e a largura da cabeça na base dos olhos (Tabela II).

Chrysoperla raimundoi apresentou as maiores medidas para todas as variáveis, e C. genanigra apresentou as menores.
Tabela II. Razão dos valores médios das mensurações realizadas na cabeça de Chrysoperla raimundoi (Freitas \& Penny, 2001), C. defreitasi (Brooks, 1994), C. genanigra (Freitas, 2003) e C. externa (Hagen, 1861) (Neuroptera, Chrysopidae).

\begin{tabular}{lcccc}
\hline Espécie & $\begin{array}{c}\text { CC/ } \\
\text { LC }\end{array}$ & $\begin{array}{c}\text { Tendência da } \\
\text { forma da } \\
\text { cabeça em vista } \\
\text { frontal }\end{array}$ & $\begin{array}{c}\text { CBO/ } \\
\text { LCI }\end{array}$ & $\begin{array}{c}\text { Tendência da } \\
\text { forma da } \\
\text { cabeça em vista } \\
\text { frontal }\end{array}$ \\
\hline C. raimundoi & 0,73 & quadrada & 0,57 & afilada \\
C. defreitasi & 0,72 & quadrada & 0,46 & arredondada? \\
C. externa & 0,75 & $\begin{array}{c}\text { retangular } \\
\text { quadrada }\end{array}$ & 0,51 & arredondada? \\
C. genanigra & 0,66 & quadredondada? & arredonda \\
\hline
\end{tabular}

$\overline{\mathrm{CC} \text { - comprimento da cabeça; LC - largura; LCI - largura da cabeça ao }}$ nível da base dos olhos; CBO - comprimento até a base dos olhos.

Apesar de a amplitude da variação para cada uma das características revelar a ocorrência de sobreposição de medidas entre as espécies, as variâncias foram baixas, e por isso o valor da moda (sempre muito próximo ao da média) mostrou-se ser um parâmetro confiável para a identificação dessas espécies.

A razão entre as medidas comprimento da cabeça e a sua largura (Tabela II), demonstrou que a forma da cabeça de $C$. externa em vista frontal tendeu para um retângulo enquanto as demais foram semelhantes a um quadrado. Já a razão entre o comprimento da distância do labro até a base dos olhos e a largura entre as bases dos olhos em C. raimundoi, evidenciou que a forma desta região tendeu a ser mais "bicuda", isto é, mais afilada distalmente, diferenciando-a das demais.

A análise das oito medidas da cabeça das quatro espécies realizadas através da análise discriminante, mostrou uma alta diferenciação entre as espécies e identificou quais medidas mais contribuíram para a diferenciação entre elas (Tabela III). O baixo valor de Wilks' Lambda $(0,055)$ indicou que as medidas separadamente poderiam discriminar as espécies, sem a necessidade de combinação entre elas. Foi possível se perceber que o comprimento da cabeça (CC), largura do vértice (LV),

Tabela III. Discriminação das variáveis da cabeça de Chrysoperla raimundoi (Freitas \& Penny, 2001), C. defreitasi (Brooks, 1994), C. genanigra (Freitas, 2003) e C. externa (Hagen, 1861) (Neuroptera, Chrysopidae) realizadas depois de uma análise de função discriminante "forward stepwise".

\begin{tabular}{lccc}
\hline & Wilks' Lambda & $\mathrm{F}$ & p-level \\
\hline CC & 0,077 & 15,39 & 0,00 \\
LC & 0,057 & 1,53 & 0,21 \\
LV & 0,068 & 8,73 & 0,00 \\
LCI & 0,060 & 3,45 & $0,02 *$ \\
CBO & 0,065 & 7,26 & 0,00 \\
CBA & 0,106 & 35,71 & 0,00 \\
LO & 0,061 & 4,13 & 0,00 \\
CO & 0,060 & 3,21 & $0,02 *$ \\
\hline
\end{tabular}

$P<0,01 ; * P<0,05$

CC - comprimento da cabeça; LC - largura; LV - largura do vértice; LCI - largura da cabeça na base dos olhos; CBA - comprimento até a base das antenas; CBO - comprimento até a base dos olhos; CO comprimento dos olhos e LO - largura dos olhos. 


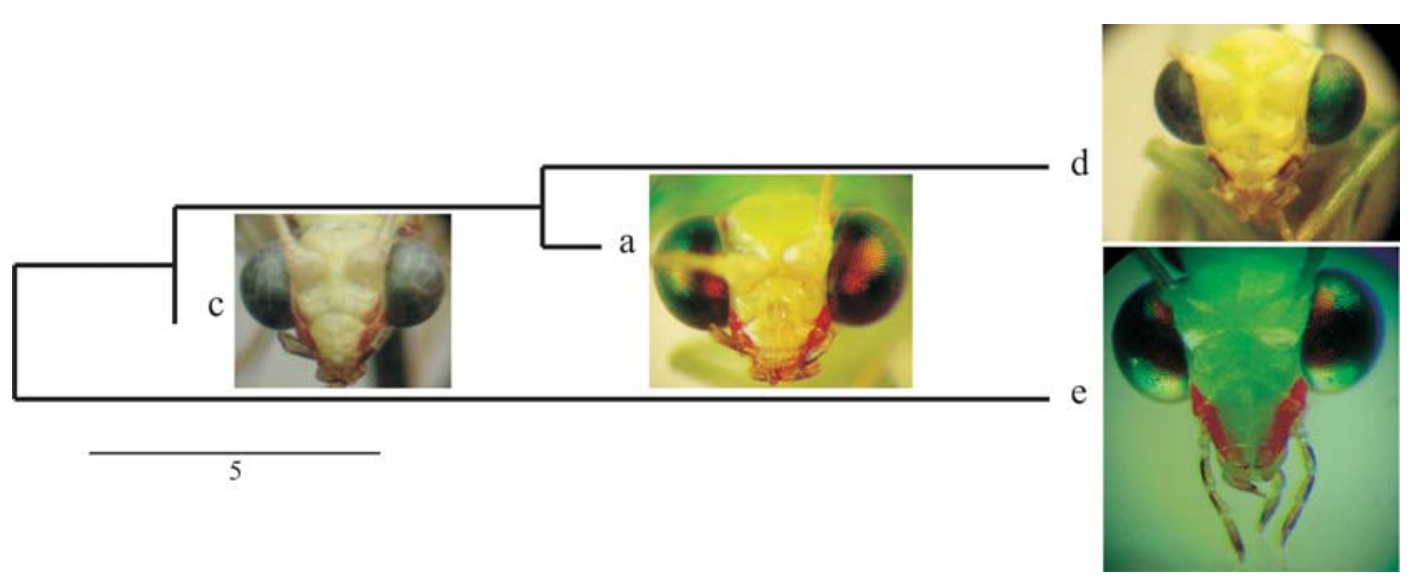

Fig. 3. Análise de similaridade através do parâmetro de distância de Mahalanobis para as quatro espécies de Chrysoperla (Neuroptera, Chrysopidae) estudadas: (e) - C. raimundoi (Freitas \& Penny, 2001); (c) - C. defreitasi (Brooks, 1994); (d) - C. genanigra (Freitas, 2003) e (a) - C. externa (Hagen, 1861).

comprimento do labro até a base dos olhos (CBO) e comprimento do labro à base da antena (CBA), foram as medidas que mais contribuíram para esta discriminação, apresentando baixo valores para Wilks' lambda e $P=0,00$ (Tabela III).

A comparação entre as quatro espécies utilizando as oito variáveis mostrou que as espécies $C$. raimundoi e C. externa obtiveram as melhores porcentagens de correta classificação com valores superiores a $90 \%$, sendo somente identificados erroneamente, duas e quatro vezes, respectivamente, como $C$. defreitasi, de acordo com a análise (Tabela IV). Já $C$. defreitasi apresentou com uma taxa de classificação de $69,4 \%$, sendo sete vezes erroneamente identificada como $C$. externa (Tabela IV).

A análise canônica mostrou que três das quatro espécies, C. raimundoi; C. genanigra e C. externa, apresentaram sobreposições entre si, mas num nível que possibilitou sua discriminação dentro do grupo. Dentre as espécies, somente C. defreitasi apresentou uma baixa resolução de acordo com esta análise, ficando sobreposta com $C$. externa e $C$. genanigra, no entanto, ela é totalmente discriminada de $C$. raimundoi (Fig. 2).

Três vetores discriminantes foram obtidos, mas a maioria da variação foi encontrada na primeira raiz ("root" 1), explicando $79 \%$ da variação presente entre estas espécies (Tabela V). As medidas que mais contribuíram estiveram relacionadas ao tamanho da cabeça e em especial ao comprimento do labro na extremidade da cabeça, até o nível dos olhos e antena.

Tabela IV. Matriz de classificação para Chrysoperla raimundoi (Freitas \& Penny, 2001), C. defreitasi (Brooks, 1994), C. genanigra (Freitas, 2003) e C. externa (Hagen, 1861) (Neuroptera, Chrysopidae).

\begin{tabular}{lccccc}
\hline & $\begin{array}{c}\text { Porcentagem } \\
(\%)\end{array}$ & $\begin{array}{c}C . \\
\text { raimundoi }\end{array}$ & $\begin{array}{c}C . \\
\text { defreitasi }\end{array}$ & $\begin{array}{c}C . \\
\text { externa }\end{array}$ & $\begin{array}{c}C . \\
\text { genanigra }\end{array}$ \\
\hline C raimundoi & 93,33 & 28 & 2 & 0 & 0 \\
C. defreitasi & 69,44 & 1 & 25 & 7 & 3 \\
C. externa & 90,47 & 0 & 4 & 38 & 0 \\
C. genanigra & 76,19 & 0 & 1 & 4 & 16 \\
\hline
\end{tabular}

Através destas medidas, foi possível se estimar a similaridade entre estas quatro espécies de ocorrência no Brasil, utilizando-se para isto o parâmetro de distância de Mahalanobis (Fig. 3). De acordo com esta análise, ficou evidente que $C$. raimundoi apresentou medidas que a diferencia das demais. Esta diferença provavelmente está relacionada ao seu perfil afilado, evidenciado pelas medidas CBO e CBA (comprimento do labro até a base dos olhos e comprimento do labro até a base das antenas, respectivamente), e ao seu grande comprimento de cabeça quando comparado com as outras três espécies.

Uma alta similaridade foi diagnosticada entre duas espécies C. genanigra e C. externa, muito embora estas possam ser diferenciadas pela presença da mancha genal de cor negra em C. genanigra e pelas menores medidas de sua cabeça, quando comparada com as outras três espécies aqui estudadas. Embora tenha ocorrido uma confluência entre as medidas de $C$. defreitasi com $C$. genanigra e $C$. externa, foi possível se observar que $C$. defreitasi foi mais similar a C. externa (Fig. 2).

A análise de similaridade morfológica (Fig. 3) também

Tabela V. Coeficientes canônicos para cada uma das variáveis morfométricas indicadas para a cabeça de Chrysoperla raimundoi (Freitas \& Penny, 2001), C. defreitasi (Brooks, 1994), C. genanigra (Freitas, 2003) e C. externa (Hagen, 1861) (Neuroptera, Chrysopidae).

\begin{tabular}{ccc}
\hline & Root 1 & Root 2 \\
\hline CC & 0,5547 & $-0,7156$ \\
LC & $-0,2667$ & $-0,0214$ \\
LV & $-0,3968$ & 0,2690 \\
LCI & $-0,2377$ & 0,3446 \\
CBO & $-0,4787$ & $-0,3253$ \\
CBA & $-0,8321$ & 0,3976 \\
LO & $-0,1144$ & $-0,4736$ \\
CO & 0,1108 & $-0,3105$ \\
\hline
\end{tabular}

CC - comprimento da cabeça; LC - largura; LV - largura do vértice; LCI - largura da cabeça na base dos olhos; CBA - comprimento até a base das antenas; CBO - comprimento até a base dos olhos; CO comprimento dos olhos e LO - largura dos olhos. 
evidenciou sua relação de proximidade morfológica com estas duas espécies, mas também as distinguiu, demostrando a existência de medidas características suficientes para diferenciá-la dentro deste grupo.

É possível que as confluências de valores apresentadas pelas espécies sejam devido ao status evolutivo ao qual estas se encontram atualmente, embora haja características determinantes morfologicamente a cada uma delas, elas ainda compartilham traços similares, produto de sua diferenciação recente e ainda em curso, o que as levam a ser caracterizadas como espécies crípticas, tal como o grupo de espécies crípticas Chrysoperla carnea onde os resultados permitiram concluir que as espécies ainda não estão totalmente discriminadas (Lourenço et al. 2006).

\section{CONCLUSÃO}

Os resultados evidenciaram que as espécies $C$. externa, C. defreitasi $C$. genanigra e $C$. raimundoi apresentam variações morfológicas intra e interespecíficas que permitem caracterizar distintamente as quatro espécies.

Em relação ao tamanho e ao comprimento, Chrysoperla raimundoi apresenta a maior cabeça e $C$. genanigra a menor.Além disto, $C$. raimundoi apresenta a região entre o labro e a base dos olhos e a largura entre as bases dos olhos "bicuda", isto é, mais afilada distalmente, diferenciando-a das demais.

A análise discriminante permitiu identificar as medidas que mais contribuíram para a diferenciação entre elas. As medidas indicadas foram, comprimento da cabeça, largura do vértice, comprimento do labro à base dos olhos e comprimento do labro à base da antena.
Agradecimentos. Nós agradecemos a Fernando de Faria Franco pelas críticas ao manuscrito e ajuda estatística. Durante este trabalho A.C.M foi bolsista pós-doc do "Programa Taxonomia" do Conselho Nacional de Desenvolvimento Científico e Tecnológico (CNPq).

\section{REFERÊNCIAS}

Adams, P. A. \& N. D. Penny. 1985. Neuroptera of the Amazon basin. Part 11a. Introduction and Chrysopini. Acta Amazonica 15: 413479.

Brooks, S. J. 1994. A taxonomic review of the common green lacewing genus Chrysoperla (Neuroptera: Chrysopidae). Bulletin of the Natural History Museum (Entomology Series) 63: 137-210.

Brooks, S. J \& P. C. Barnard. 1990. The green lacewings of the world: a generic review (Neuroptera: Chrysopidae). Bulletin of the Natural History Museum (Entomology Series) 59: 117-286.

Freitas, S. 2003. Chrysoperla Steinmann, 1964 (Neuroptera: Chrysopidae): descrição de uma nova espécie do Brasil. Revista Brasileira de Entomologia 47: 385-387.

Freitas, S. \& N. D. Penny. 2001. The green lacewings (Neuroptera: Chrysopidae) of brazilian agro-ecosystems. Proceedings of the California Academy of Sciences 52: 245-395.

Hagen, H. 1861. Synopsis of the Neuroptera of North America, with a list of the South American species. Smithsonian Miscellaneous Collections 49: 1-347.

Kumar, S.; K. Tamura \& M. Nei. 2004. MEGA 3: Integrated software for Molecular Evolutionary Genetics Analysis and sequence alignment. Briefings in Bioinformatics 5: 150-163.

Lourenço, P.; C. Brito; T. Backeljau; D. Thierry \& M. A. Ventura. 2006. Molecular systematics of the Chrysoperla carnea group (Neuroptera: Chrysopidae) in Europe. Journal of Zoological Systematics and Evolutionary Research 44: 180-184.

Statistica v. 6. StatSoft, Inc. (2001). STATISTICA (data analysis software system), www.statsoft.com.

Stelzl, M.; D. Devetak. 1999. Neuroptera in agricultural ecosystems. Agriculture Ecosystems \& Environment 74: 305-321.

Tauber, M. J.; C. A. Tauber.; K. M. Daane \& K. S. Hagen. 2000. Commercialization of predators: recent lessons from green lacewings (Neuroptera: Chrysopidae). American Entomologist 46: 26-38. 